\title{
Outcome and Safety of Cytoreductive Surgery (CRS) and Hyperthermic Intraperitoneal Chemotherapy (HIPEC) +/- Intraoperative Radiation Therapy (IORT) in the Management of Peritoneal Sarcomatosis: A Real-World Experience
}

\author{
Ahmed Elashwah ${ }^{1,2}$, Ahmed Badran ${ }^{3,4}$, Mahmoud A. Elshenawy ${ }^{3,5}$, Ayman Azzam \\ 6, 7 , Rania Naguib ${ }^{8,9}$, Aisha Alshibani ${ }^{10}$, Reem Alrakaf ${ }^{6}$, Abdelmoniem Eldali ${ }^{11}$, \\ Tarek Amin ${ }^{6}$
}

${ }^{1}$ Radiation Oncology Department, Oncology Center, King Faisal Specialist Hospital and Research Center, Riyadh, Saudi Arabia; ${ }^{2}$ Kasr Al-Aini Center for Clinical Oncology and Nuclear Medicine (NEMROCK), Faculty of Medicine, Cairo University, Cairo, Egypt; ${ }^{3}$ Medical Oncology Department, Oncology Center, King Faisal Specialist Hospital and Research Center, Riyadh, Saudi Arabia; ${ }^{4}$ Clinical Oncology Department, Faculty of Medicine, Ain Shams University, Cairo, Egypt; ${ }^{5}$ Clinical Oncology and Nuclear Medicine Department, Faculty of Medicine, Menoufia University, Shebin El-Kom, Egypt; ${ }^{6}$ Surgical Oncology Department, Oncology Center, King Faisal Specialist Hospital and Research Center, Riyadh, Saudi Arabia; ${ }^{7}$ General Surgery Department, Faculty of Medicine, Alexandria University, Alexandria, Egypt; ${ }^{8}$ Internal Medicine Department, Faculty of Medicine, Alexandria University, Alexandria, Egypt; ${ }^{9}$ Clinical Science Department, College of Medicine, Princess Nourah Bint Abdulrahman University, Riyadh, Saudi Arabia; ${ }^{10}$ Internal Medicine Department, King Faisal Specialist Hospital and Research Center, Riyadh, Saudi Arabia; 11 Department of Biostatistics, Epidemiology and Scientific Computing, King Faisal Specialist Hospital and Research Center, Riyadh, Saudi Arabia

\begin{abstract}
Background: Peritoneal sarcomatosis (PS) is an aggressive disease; cytoreductive surgery (CRS) could be curative. Aim: Can the addition of hyperthermic intraperitoneal chemotherapy (HIPEC) +/- intraoperative radiation therapy (IORT) overcome treatment failure with an overall survival benefit.

Methods: Retrospective review of the medical records of patients with PS treated by CRS, HIPEC and IORT at a comprehensive cancer center in the period between 2011-2016.

Results: Twenty-four patients were identified. Fifteen were men and their median age was 58 years. Liposarcoma was the most frequent diagnosis (50\%). Cytoreduction completeness (CC) score 0/1 was achieved in 19 patients, with a median pathological peritoneal cancer index (pPCI) of 12. Intraoperative radiation therapy was given in 16 patients. Eight patients developed grade III-IV Clavien-Dindo post-operative complications and 1 patient died 5 days post operative. Adjuvant chemotherapy was received in 9 patients. After a median follow-up of 28.5 months, the median PFS was 20.7 months, while the estimated 2- and 4-year PFS were $37.1 \%$ and $19.1 \%$, respectively. The median OS was 176.5 months and the estimated 2- and 4-year OS were $95.8 \%$ and $79.8 \%$, respectively. In the univariate analysis, the PFS differed significantly according to the CC score only. The median PFS for patients with CC 0-1 was 23.8 vs. 8.8 months for those with CC $2-3$ ( $p=0.027$ ).

Conclusions: The addition of HIPEC and IORT to CRS in the management of PS is feasible and safe. Comparing our results to several studies, this multimodality approach seems to improve local and regional control rates. A larger cohort of patients is needed for further evaluation and to give a concrete conclusion.
\end{abstract}

Keywords: Peritoneal sarcomatosis, Cytoreductive Surgery, Hyperthermic intraperitoneal chemotherapy, Intraoperative radiation therapy 
Corresponding author: Ahmed Elashwah, MD; Radiation Oncology Department, Oncology Center, King Faisal Specialist Hospital and Research Center, Riyadh, Saudi Arabia; Email: ashwah a80@yahoo.com

Submitted: 2-October-2021, Revised: 12-December-2021, Accepted: 21-December-2021, Published online: 11-February-2022

(cc) BY

\section{Introduction}

Soft tissue sarcomas represent about $1 \%$ of all adult malignancies, a third of them originate from the abdominal viscera or retroperitoneum ${ }^{1}$. They are characterized by the high ability for hematogenous spread, typically to the lungs, liver, and direct spread to involve other peritoneal surfaces and adjacent organs ${ }^{2}$. They had also; a high post-surgical locoregional failure rate ranged from 35 to $82 \%^{3,4}$.

Peritoneal sarcomatosis (PS) presents a diffuse form of intra-abdominal dissemination; either due to recurrence or spread by seeding to the nearby peritoneal surface. It could be the initial diagnosis, but more frequently occurs at recurrence, most probably as a result of tumor spillage during the initial resection ${ }^{5-7}$. The prognosis of patients with PS is poor, therefore the ultimate need for research to find the best treatment options increased ${ }^{7}$.

Cytoreductive surgery (CRS) with hyperthermic intraperitoneal chemotherapy (HIPEC) has achieved prolonged survival in patients with peritoneal surface disease from a variety of epithelial tumors of appendicular, colorectal, and mesothelioma origins 8-11.

There is great controversy about the use of CRSHIPEC in PS patients, probably due to the high ability of sarcoma for hematogenous spread and the lack of effective chemotherapeutic agents ${ }^{4,5,12-18}$.

Intraoperative radiation therapy (IORT) is a highly conformal radiation therapy modality that is administered in the operating theater. It has been used in a variety of malignancies, including retroperitoneal sarcoma, in order to increase the tumor radiation dose without exceeding normal tissue tolerance doses for better tumor local control $19-23$

Many studies have addressed the prognostic value of many factors such as: KI-67 index, pretreatment inflammatory markers such as neutrophil-lymphocyte ratio (NLR) and plateletlymphocyte ratio (PLR) in soft tissue sarcoma patients reporting that patients with high levels of these markers have poor prognosis and survival ${ }^{24}$. 27.

In this study, we reviewed the outcome of PS patients who had been treated with CRS-HIPEC +/-
IORT, including postoperative morbidity and mortality, local control rate, progression-free survival (PFS), overall survival (OS) and the significant correlation of multiple variables including the KI-67 index, NLR and PLR with survival.

\section{Methods}

The medical records of 24 PS patients treated with the multimodal approach including (CRS and HIPEC +/- IORT) at the King Faisal Specialized Hospital and Research Center, Riyadh, Saudi Arabia, in the period between January 2011 and December 2016 were retrospectively reviewed. The data collected included age, sex, body mass index, Eastern Cooperative Oncology Group (ECOG) performance status, histopathological subtype, histological grade, ki-67 index (number of KI-67 positive tumor cells), initial NLR, initial PLR, American Joint Committee on Cancer (AJCC) stage, preoperative tumor size, preoperative treatment received (chemotherapy or radiation therapy), operational details (including completeness of cytoreduction [CC], pathological peritoneal cancer index [pPCI] and chemotherapeutic agent used in HIPEC), the dose of IORT, postoperative complications according to the Clavien-Dindo classification system, postoperative treatment received, pattern of disease recurrence (locoregional, distant or combined) and current status of patient (alive with a disease or alive without disease or dead).

The HIPEC technique is used in our center in addition to CRS in the management of PS patients with the following inclusion criteria: (1) ECOG performance status $\leq 2$, (2) satisfactory laboratory work, (3) proven diagnosis of PS confirmed by preoperative biopsy, (4) no evidence of distant extra abdominopelvic metastases to the liver, lungs, brain or bones. The details of the operative and HIPEC technique were published earlier in our previous studies ${ }^{17,28}$.

After completion of the surgical procedure, residual tumor assessment was performed intraoperatively using the standard CC scores, as documented by Sugarbaker, CC-0 (no gross residual disease) was regarded as complete cytoreduction, whereas CC-1 (up to $2.5 \mathrm{~mm}$ gross residual disease) 
was regarded as near-complete cytoreduction ${ }^{29}$. Intraoperative radiation therapy is usually used in our center for patients with PS using Mobetron ${ }^{\circledR}$ in a dose range of 10-20 Gy. The total dose of IORT received depended on the extent of the residual tumor after resection. IORT was used in patients with CC- 0 and $\mathrm{CC}-1$ only. In patients with $\mathrm{CC}-0$, a dose of 10-12 Gy is used. For the patients CC-1(with residual tumors less than $1 \mathrm{~mm}$ ), a dose of $12-15$ Gy is used. For those with CC-1(residual tumor 1-2.5 $\mathrm{mm}$ ), a dose of 15 to $20 \mathrm{~Gy}$. A dose between 10 and 20 Gy was considered a safe dose with minimal postoperative side effects 30,31 , taking in consideration other factors as the location of nearby risk structures and the dose of previous radiation therapy if present.

Many therapeutic agents for HIPEC were used as a combination of cisplatin $\left(50 \mathrm{mg} / \mathrm{m}^{2}\right)$ plus doxorubicin $\left(15 \mathrm{mg} / \mathrm{m}^{2}\right)$ infused over 90 minutes or single agent melphalan $\left(60 \mathrm{mg} / \mathrm{m}^{2}\right)$ infused over 60 minutes. The choice of HIPEC therapeutic agent depends on the case as agreed by the multidisciplinary medical oncology and surgical oncology team treating.

During the HIPEC procedure, all hemodynamic and cardiopulmonary parameters were strictly monitored. After completion of the entire procedure, including CRS, HIPEC +/- IORT, all patients were transferred to the intensive care unit (ICU) for 1 to 3 days (median:1 day) and then transferred to the surgical ward for recovery.

Postoperative complications were evaluated according to the Clavien-Dindo grading system (Table 1) ${ }^{32}$.

In some cases, adjuvant treatment (chemotherapy, radiation therapy, or both) was planned if deemed indicated based on the postoperative pathological and radiological data.

All patients were kept at regular follow-up, every 3 months during the first 2 years after HIPEC, every 6 months for another 2 years, then annually. Followup investigations included complete blood work, chest, abdomen, and pelvis CT, abdominal magnetic resonance imaging + / - PET-CT (when indicated).

\section{Statistical analysis}

Statistical analysis was performed using IBM SPSS ${ }^{\circledR}$ Statistics version 26 (IBM ${ }^{\circledR}$ Corp., Armonk, NY, USA). Quantitative variables were described as median and range. Qualitative variables were described as numbers and percentages. The study's primary endpoints were toxicity profile (assessed according to the Clavien-Dindo [CD] grading system) and PFS. The secondary endpoint was OS. Progression-free survival was calculated from the date of surgery to the date of documented progression, death, or the last follow up; and OS from the date of diagnosis to the date of death or the last follow-up. Survival analysis was performed using the Kaplan-Meier method and survival curves were compared using the log-rank test. A $p$ value $<0.05$ was considered statistically significant.

\section{Table 1: The Clavien-Dindo grading system of postoperative complications}

\begin{tabular}{|c|c|}
\hline Grade & Definition \\
\hline Grade 1 & $\begin{array}{l}\text { Any deviation from the normal } \\
\text { postoperative course without the } \\
\text { need for pharmacological treatment } \\
\text { or surgical, endoscopic, and } \\
\text { radiological interventions. } \\
\text { Acceptable therapeutic regimens are } \\
\text { drugs as antiemetics, antipyretics, } \\
\text { analgesics, diuretics and electrolytes, } \\
\text { and physiotherapy. This grade also } \\
\text { includes wound infections opened at } \\
\text { the bedside. }\end{array}$ \\
\hline Grade II & $\begin{array}{l}\text { Requiring pharmacological } \\
\text { treatment with drugs other than } \\
\text { such allowed for grade I } \\
\text { complications. Blood transfusions } \\
\text { and total parenteral nutrition are } \\
\text { also included. }\end{array}$ \\
\hline Grade III & $\begin{array}{l}\text { Requiring surgical, endoscopic, or } \\
\text { radiological intervention. }\end{array}$ \\
\hline Grade III-a & $\begin{array}{l}\text { Intervention not under general } \\
\text { anesthesia. }\end{array}$ \\
\hline Grade III-b & $\begin{array}{l}\text { Intervention under general } \\
\text { anesthesia. }\end{array}$ \\
\hline Grade IV & $\begin{array}{l}\text { Life-threatening complications } \\
\text { (including CNS complications) } \\
\text { requiring IC/ICU-management. }\end{array}$ \\
\hline Grade IV-a & $\begin{array}{l}\text { Single organ dysfunction (including } \\
\text { dialysis). }\end{array}$ \\
\hline Grade IV-b & Multi-organ dysfunction. \\
\hline Grade V & Death of the patient. \\
\hline
\end{tabular}

\section{Results}

Fifteen males and nine females were reviewed, the median age at the time of CRS was 58 (31-77) years. Seventeen (71\%) patients had stage III disease according to AJCC staging system. KI-67 were detected in $14(58 \%)$ patients with a variable range of 6-370/10 high power field (HPF), with a median value of 56/10 HPF. Different neoadjuvant chemotherapy protocols were used in $5(20.8 \%)$ patients; (imatinib, doxorubicin and combined 
ifosfamide-etoposide). Three patients received neoadjuvant radiation therapy with a dose of 45Gy/25 fractions using intensity modulated radiation therapy technique. Detailed patients and treatment characteristics are listed in Table 2.

Table 2: Baseline characteristics of 24 patients with peritoneal sarcomatosis and their pre-surgical treatment

\begin{tabular}{ll}
\hline Characteristic & Description \\
\hline $\begin{array}{l}\text { Age in years at CRS time (median } \\
\text { [range]) }\end{array}$ & $58(31-77)$ \\
\hline Gender & \\
\hline Male & $15(62.5 \%)$ \\
\hline Female & $9(37.5 \%)$ \\
\hline ECOG performance status & $14(58 \%)$ \\
\hline 1 & $10(42 \%)$ \\
\hline 2 & \\
\hline Histopathological subtypes & $12(50 \%)$ \\
\hline Liposarcoma & $4(16.7 \%)$ \\
\hline Leiomyosarcoma & $3(12.5 \%)$ \\
\hline Gastrointestinal stromal tumor & $5(20.8 \%)$ \\
\hline Others & \\
\hline Histopathological grades & $7(29 \%)$ \\
\hline G1 & $9(37.5 \%)$ \\
\hline G2 & $8(33.5 \%)$ \\
\hline G3 & $14(58 \%)$ \\
\hline KI-67 positive tumor cells (KI-67 index) & $56(6-370 / 10$ \\
\hline KI-67 index (median [range]) & HPF) \\
\hline Pre-operative tumor size in cm (median & $12(6-21)$ \\
\hline range]) & \\
\hline AJCC Stage & $7(29 \%)$ \\
\hline I & $6(25 \%)$ \\
\hline IIIa & $11(46 \%)$ \\
\hline IIIb & $5(20.8 \%)$ \\
\hline Neoadjuvant chemotherapy & $19(79.2 \%)$ \\
\hline Given & $21(87.5 \%)$ \\
\hline Not given & \\
\hline Neoadjuvant radiation therapy & \\
\hline Given & \\
\hline Not given & \\
\hline CCoG: Eastern Cooperative Oncology Group; AJcc: American Joint \\
\hline
\end{tabular}

All patients underwent CRS and HIPEC to achieve cure. The CC score (0/1) was achieved in 19 (79.17\%) patients with a median pPCI of 12 (range: 3 to 28). Melphalan was the most used chemotherapeutic agent in HIPEC, it was used in 16 (66.67\%) patients. IORT was given in $16(66.67 \%)$ patients (dose range 10-15 Gy), Table 3.

Different adjuvant chemotherapy protocols were used in $9(37.5 \%)$ patients including (imatinib,
Table 3: Summary of surgery outcome, HIPEC, IORT, and postoperative treatment

\begin{tabular}{ll}
\hline \multicolumn{1}{l}{ Treatment / outcome } & Description \\
\hline \begin{tabular}{l} 
Cytoreduction completeness (CC) score \\
\hline 0
\end{tabular} & $12(50 \%)$ \\
\hline 1 & $7(29.2 \%)$ \\
\hline 3 & $4(16.7 \%)$ \\
\hline $\begin{array}{l}\text { Pathological peritoneal cancer index } \\
\text { (pPCI) }\end{array}$ & $1(4.1 \%)$ \\
\hline Median & 12 \\
\hline$\leq 10$ & $10(41.6 \%)$ \\
\hline $10-20$ & $9(37.5 \%)$ \\
\hline$>20$ & $5(20.9 \%)$ \\
\hline Chemotherapeutic agents used in HIPEC \\
\hline Melphalan & $16(66.6 \%)$ \\
\hline Cisplatin/doxorubicin & $8(33.3 \%)$ \\
\hline IORT received & $16(66.6 \%)$ \\
\hline IORT dose & $6(25 \%)$ \\
\hline 1000 cGy & $2(8.3 \%)$ \\
\hline 1200 cGy & $2(8.3 \%)$ \\
\hline 1250 cGy & $6(25 \%)$ \\
\hline 1500 cGy & $9(37.5 \%)$ \\
\hline Adjuvant chemotherapy received & \\
\hline
\end{tabular}

HIPEC: Hyperthermic intraperitoneal chemotherapy, IORT: Intraoperative radiation therapy

doxorubicin, and ifosfamide, single agent doxorubicin and single agent gemcitabine).

Grade I CD complications occurred in 6 (25\%) patients and grade II in 9 (37.5\%). Nine (37.5\%) patients developed $\geq$ grade III Clavien-Dindo (CD) complications, and 1 (4.2\%) patient died (grade V CD) 5 days after operation in the ICU due to massive pulmonary embolism despite full coverage of prophylactic anticoagulants. The details of grade III and IV CD complications are illustrated in Table 4.

After a median follow up of 28.5 (2-70) months, the median overall (locoregional and systemic) PFS was 20.7 months, while the estimated 2 and 4 years overall PFS were $37.1 \%$ and $19.1 \%$, respectively (Figure 1).

Fifteen $(62.5 \%)$ patients developed systemic progression (two of them developed both locoregional and systemic progression). The lung was the site most affected at the time of progression in $8 / 15(53.3 \%)$ patients. Isolated locoregional progression occurred in two (8.3\%) patients. The 2 cases with isolated local progression underwent redo CRS and HIPEC with an average of 36 months between the first and redo surgery. The estimated 2and 4-year locoregional PFS were $88.4 \%$ and $60.6 \%$, respectively, while the estimated 2 and 4 years systemic PFS were $37.1 \%$ and $27.8 \%$, respectively. 
The median OS was 176.5 months, with estimated 2- and 4-year OS were $95.8 \%$ and $79.8 \%$ respectively (Figure 2).

For PFS, in the univariate analysis, the CC score was significantly correlated with PFS as the median PFS for patients with CC $0-1$ was 23.8 months vs 8.8 months for those with CC $2-3$ ( $p=0.027$ ). Progressionfree survival did not differ significantly according to the other variables studied (Table 5). Similarly, none of the studied variables was associated with a significant difference in OS in the univariate analysis.

Table 4: Management and outcome of grade III and IV Clavein Dindo (CD) complications

\begin{tabular}{llllr}
\hline CD Grade & n. (\%) & Complication & Management & Outcome \\
\hline III a & $2(8.3)$ & Pleural effusion & Pleurocentesis & Recovered \\
\cline { 2 - 5 } & $1(4.2)$ & Pancreatic fistula & Ultrasound-guided drainage & Recovered \\
\cline { 2 - 5 } & $1(4.2)$ & Urinary bladder fistula & Cystoscopy and Foley's catheter insertion & Recovered \\
\hline III b & $1(4.2)$ & Bowel leakage & Exploration & Recovered \\
\cline { 2 - 5 } & $1(4.2)$ & Bleeding & Exploration and hematoma evacuation & Recovered \\
\cline { 2 - 5 } & $1(4.2)$ & Wound dehiscence & Debridement and flap & Recovered \\
\hline IV a & $1(4.2)$ & Respiratory failure & Resuscitation in the intensive care unit & Recovered \\
\hline
\end{tabular}

Table 5: univariate analysis correlation of multiple parameters with progression-free and overall survival

\begin{tabular}{|c|c|c|c|c|}
\hline Survival & Parameter & & $\begin{array}{l}\text { Estimated median } \\
\text { survival (months) }\end{array}$ & $p$ value \\
\hline \multirow{10}{*}{$\begin{array}{l}\text { Progression-free } \\
\text { survival }\end{array}$} & \multirow[t]{2}{*}{ Cytoreduction completeness (CC) score } & 0,1 & 23.8 & \multirow[t]{2}{*}{0.027} \\
\hline & & 2,3 & 8.8 & \\
\hline & \multirow[t]{3}{*}{ Pathological peritoneal cancer index (pPCI) } & $\leq 10$ & 23.9 & \multirow[t]{3}{*}{0.382} \\
\hline & & $10-20$ & 20.2 & \\
\hline & & $>20$ & 8.4 & \\
\hline & \multirow[t]{3}{*}{ Histopathological grade } & G1 & 22.6 & \multirow[t]{3}{*}{0.316} \\
\hline & & G2 & 19.4 & \\
\hline & & G3 & 8.5 & \\
\hline & \multirow[t]{2}{*}{ Tumor size $(\mathrm{cm})$} & $\leq 11$ & 21.9 & \multirow[t]{2}{*}{0624} \\
\hline & & $>11$ & 14.4 & \\
\hline \multirow[t]{9}{*}{ Overall survival } & \multirow[t]{2}{*}{ KI-67 index (/10 HPF) } & $\leq 50$ & 181.8 & \multirow[t]{2}{*}{0.324} \\
\hline & & $>50$ & 155 & \\
\hline & \multirow[t]{2}{*}{ Neutrophil-lymphocyte ratio (NLR) } & $\leq 2.5$ & 176 & \multirow[t]{2}{*}{0.805} \\
\hline & & $>2.5$ & 104 & \\
\hline & \multirow[t]{2}{*}{ Platelet-lymphocyte ratio (PLR) } & $\leq 135$ & 180 & \multirow[t]{2}{*}{0.423} \\
\hline & & $>135$ & 124 & \\
\hline & \multirow{3}{*}{$\begin{array}{l}\text { American Joint Committee on Cancer (AJCC) } \\
\text { stage }\end{array}$} & $\mathrm{I}$ & 184 & \multirow[t]{3}{*}{0.235} \\
\hline & & III a & 160 & \\
\hline & & III b & 87 & \\
\hline
\end{tabular}

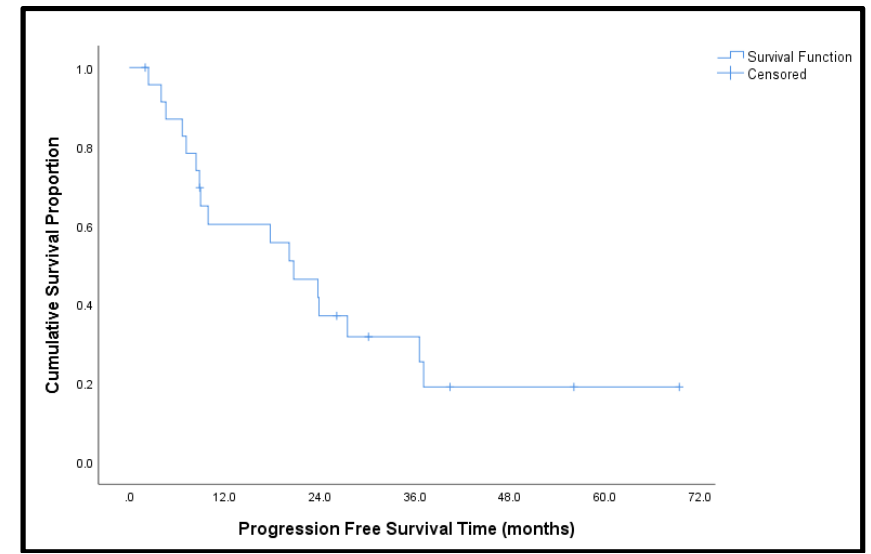

Figure 1: Kaplan-Meier curve of progression-free survival

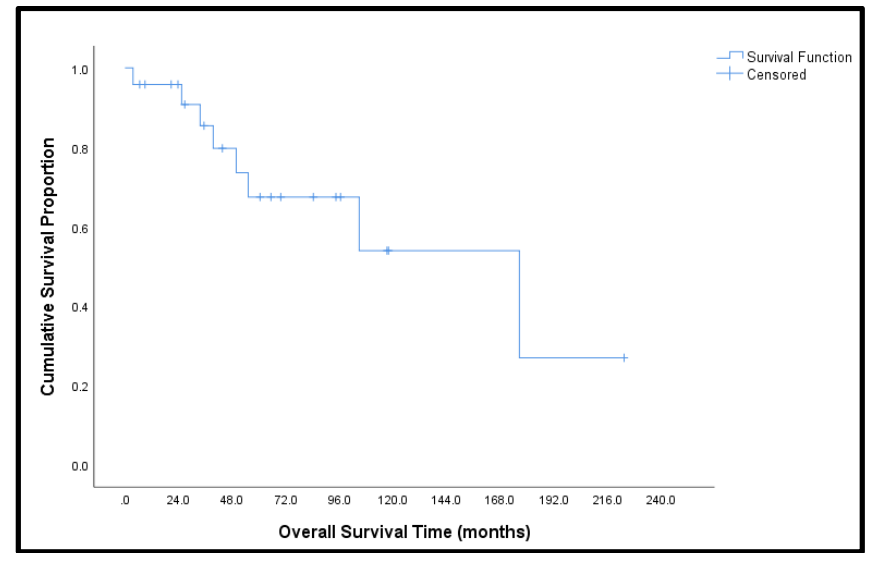

Figure 2: Kaplan-Meier curve of overall survival 


\section{Discussion}

The theoretical advantage of using HIPEC after major CRS in patients with PS may come from the ability to achieve high regional concentrations of chemotherapeutic agents while keeping systemic drug levels low. This is mostly due to the slow movement of drugs from the peritoneal cavity into the plasma (peritoneal clearance) as an effect of the peritoneal-plasma barrier ${ }^{33,34}$. The other advantage is the beneficial exposure of potential hepatic micrometastases to chemotherapeutic agents as the blood drainage of the peritoneal surface reaches the liver via the portal vein ${ }^{35}$.

The benefit of using HIPEC with CRS has been thoroughly studied in many patients with peritoneal surface involvement from many cancers of epithelial origin resulting in improvement of the locoregional control ${ }^{36-42}$, however, these benefits of using HIPEC in addition to CRS in PS have not been documented in many studies ${ }^{4,5,12-18}$. In the current study, we have better median os (176.5 months) in comparison to those patients in a study performed by Rossi et al ${ }^{4}$, where the median OS was 34 months, Baratti et al ${ }^{43}$ with reported median OS of 26.2 months and in Lim et al ${ }^{5}$ study, where the median OS was 16.9 months. In the current study we have a better median PFS and OS (20.7 and 176.5 months, respectively) compared to patients enrolled in the study of Karamveri et al. ${ }^{18}$, where the median PFS and OS were 9 and 55 months, respectively. That survival improvement may come from the improved locoregional control (will be discussed in a separate section below) in our study compared to these studies. Despite improved survival in our study compared to other studies, strong recommendations cannot be concluded due to the great difference in histopathology, pPCI, and degree of cytoreduction between these studies.

The debate of using HIPEC in addition to CRS in PS could be related to the high ability of sarcomas for hematogenous spread as, in one series, $11 \%$ of PS patients had distant dissemination at presentation increased to $28 \%$ during the treatment course ${ }^{6}$. Another series reported that distant metastasis could involve multiple organs, most commonly lung in $16 \%$ of cases followed by liver in $11 \%$ of cases ${ }^{43}$. This was also evident in our study where 15 (62.5\%) patients developed systemic recurrence and two (8.3\%) patients developed only isolated local recurrence.

In trials to reduce the incidence of distant metastasis post-CRS and HIPEC, many investigators have used a bidirectional intraoperative intravenous chemotherapy in combination with HIPEC in patients with peritoneal metastasis from a variety of neoplasms including gastric and colorectal malignancies with promising results ${ }^{44,45}$, however, no published data about its efficacy in PS. A recently published study conducted at our center by Hakeam et al ${ }^{46}$ was assessing the safety and reported side effects of bidirectional intraoperative intravenous chemotherapy using iphosphamide in combination with HIPEC in 18 patients with peritoneal metastasis from different primaries ( $50 \%$ of the patients had PS). They concluded that bidirectional intraoperative intravenous chemotherapy in combination with HIPEC was generally tolerable with low rates of mild leukopenia and frequent mild thrombocytopenia, but severe suppression of platelets was uncommon. They reported nephrotoxicity in one-third of the patients. Survival data for the patients included in this study are still pending.

When comparing the locoregional recurrence rate in our study (16.6\%) with other studies, Karamveri et al. ${ }^{18}$ showed locoregional recurrences in $65.5 \%$ of patients, Lim et al. 5 study, the locoregional recurrence rates were $79 \%$ in patients treated with cisplatin and $68 \%$ in the group of patients treated with combination cisplatin/mitoxantrone in HIPEC regimens. In Baratti et al ${ }^{43}$ study, the isolated local recurrence rate was $57.1 \%$ and in Rossi et al ${ }^{4}$ study, the local recurrence rate was $67 \%$. The improved locoregional control rate in our study in comparison to the above-mentioned studies came despite that our patients had advanced disease. This is evidenced in our study by the mean PPCI of 13.6 and pPCI $>10$ in $14(58.3 \%)$ patients in comparison to patients enrolled in Karamveri et al ${ }^{18}$ study where $31 \%$ had PCI $>6$ and in Rossi et al ${ }^{4}$ study where the mean PCI was 7 . The improved local regional control rate in our study could be explained by using IORT (used in 16 patients), the different agents used in HIPEC (melphalan was used in most of our patients (66.6\%), in addition to highly experienced surgeons with highly efficient skills.

In the univariate analysis, the $\mathrm{CC}$ score was significantly correlated with PFS ( $p=0.027)$. Patients with $\mathrm{pPCI}>10$ had a lower median PFS compared to those with pPCI $<10$ although it was statistically nonsignificant. These results were compatible with other studies conducted by Rossi et al ${ }^{4}$, Reese et al ${ }^{15}$, and Naffouje et al ${ }^{47}$; where both CC and PCI are directly correlated with survival. 
The extent of PS was evaluated intraoperatively using the peritoneal cancer index (PCI) score ${ }^{48}$. Because surgical PCI (sPCI) is calculated based on a subjective evaluation of the extent of peritoneal disease during surgery, which often results in an overestimation of the score, we started to use pathological PCI (pPCI), which may be a more accurate and objective method for determining PCI and evaluating the extent of peritoneal disease ${ }^{49}$. This may have a more prognostic significance ${ }^{50}$.

In our study, many other factors (including KI-67 index, tumor size, histopathological grade and stage) were associated with better either PFS or OS although none of them had statistically significant correlation with PFS or OS in spite of being significantly correlated with PFS and OS in many other studies ${ }^{24,51}$, for example, in our study, patients with high initial level of NLR and PLR have a lower median OS compared to those patients with lower levels. However, the relation was statistically nonsignificant. These findings are comparable to results from other studies where a high level of NLR and PLR were significantly correlating with survival 25-27. In our study, histological subtypes were not significantly correlated with survival. These findings are contradictory to the study conducted by Baratti et al. ${ }^{43}$ where the histological subtype was significantly correlated with survival, as retroperitoneal liposarcoma had the best os (median 34 months) but with $100 \%$ peritoneal relapse. This difference could be attributed to the small number of patients enrolled in our study or to a different cohort of patients.

Postoperative morbidity and mortality in our study were assessed using the Clavien-Dindo grading system ${ }^{32}$. The 30 days mortality rate was $4.1 \%$, with 8 (33.3\%) patients developed grade III-IV complications (only 3 patients developed grade IIIb complications requiring intervention under general anesthesia). Karamveri et al 18 reported a postoperative mortality and morbidity rate of $0 \%$ and $20.7 \%$ respectively, and grade III, IV complications occurred in $13.8 \%$ of patients. In Baratti et al. ${ }^{43}$ study, the operative mortality and morbidity were $3.7 \%$ and $21.6 \%$ respectively, while in Rossi et al. ${ }^{4}$ study, the morbidity rate was $33 \%$ and the moderate to severe locoregional toxicity rate was $15 \%$.

Multiple studies have addressed the value of using IORT in retroperitoneal sarcoma because it is difficult to completely remove with negative margin (due to its usual large size and proximity to critical structures) in addition to challenging delivery of an adequate dose of external beam radiation therapy (EBRT) postoperatively ${ }^{19-23}$. A randomized trial conducted by Sindelar et al. ${ }^{23}$ reported a better local control rate with IORT in addition to low-dose postoperative EBRT compared to high-dose postoperative EBRT alone in patients with retroperitoneal sarcoma ( $60 \%$ vs. $20 \%, p<0.05)$ after a median follow-up of 8 years.

Although some previous studies had addressed the benefits of combining both HIPEC and IORT with CRS in many cancer types with proved peritoneal involvement with documented improvement in locoregional control ${ }^{31,52-55}$, yet to our mind this is the first study constructed initially to assess the benefit of combining both HIPEC and IORT with CRS in locoregional control of PS. In our study, we used IORT in addition to HIPEC in $66.6 \%$ of the patients taking into account the factors mentioned above that make complete surgical excision with a negative margin difficult, resulting in local control impairment. The sites, doses, and parameters of applied IORT were chosen based on the clinical judgment and discussion between the treating surgeon and radiation oncologist according to the actual findings in the operative room.

The limitations in our study include the wide variation in histopathological subtypes, treatment protocol used (neoadjuvant/ adjuvant chemotherapy, neoadjuvant radiation therapy, in addition to the different chemotherapeutic agents used in HIPEC), this diversity might be due to the rarity of such disease with few patient numbers.

\section{Conclusions}

Addition of HIPEC and IORT to CRS in the management of PS is feasible and safe. Comparing our results with several recent studies, this multimodal approach appears to improve local and regional control rates. A larger cohort of patients is needed for further evaluation and to give a concrete conclusion.

\section{Acknowledgments \\ None. \\ Authors' contribution \\ Conception or design: AE; Acquisition, analysis or interpretation of data: $\mathrm{AE}, \mathrm{AB}, \mathrm{MAE}, \mathrm{AA}, \mathrm{RA}, \mathrm{AbE}$; Drafting or revising the manuscript: $\mathrm{AE}, \mathrm{AA}, \mathrm{RN}, \mathrm{TA}$; Approval of the manuscript version to be published: All authors; Agreement to be accountable for all aspects of the work: All authors.}

\section{Conflict of interest}

The authors declare that they have no conflict of interest to disclose.

Data a vailability 
Deidentified individual participant data used to produce the results of this study are available from the corresponding author (AE) on request.

\section{Ethical considerations}

This research has been approved by the Research Advisory Council (RAC) of the King Faisal Specialist Hospital and Research Center, Riyadh, Saudi Arabia (RAC Project \#2181187)

\section{Funding}

The authors did not receive funding for this study.

\section{Study registration}

None.

\section{References}

1. Torre LA, Siegel RL, Ward EM, Jemal A. Global cancer incidence and mortality rates and trends--An update. Cancer epidemiol Biomarkers Prev. 2016; 25(1): 1627.

2. Baldini EH, Halperin EC, Wazer DE, et al.. Soft Tissue Sarcoma. Perez and Brady's Principles and Practice of Radiation Oncology. 6th ed. Philadelphia: Lippincott Williams \& Wilkins, 2013, p.1618.

3. Mudan SS, Conlon KC, Woodruff JM, Lewis JJ, Brennan MF. Salvage surgery for patients with recurrent gastrointestinal sarcoma: prognostic factors to guide patient selection. Cancer. 2000; 88(1): 66-74.

4. Rossi CR, Deraco M, De Simone $M$, et al. Hyperthermic intraperitoneal intraoperative chemotherapy after cytoreductive surgery for the treatment of abdominal sarcomatosis: clinical outcome and prognostic factors in 60 consecutive patients. Cancer. 2004; 100(9): 1943-1950.

5. Lim SJ, Cormier JN, Feig BW, et al. Toxicity and outcomes associated with surgical cytoreduction and hyperthermic intraperitoneal chemotherapy (HIPEC) for patients with sarcomatosis. Ann Surg Oncol. 2007; 14(8): 2309-2318.

6. Karakousis CP, Blumenson LE, Canavese G, Rao U. Surgery for disseminated abdominal sarcoma. Am J Surg. 1992; 163(6): 560-564.

7. Bilimoria MM, Holtz DJ, Mirza NQ, et al. Tumor volume as a prognostic factor for sarcomatosis. Cancer. 2002; 94(9): 2441-2446.

8. Levine EA, Stewart JH 4th, Russell GB, Geisinger KR, Loggie BL, Shen P. Cytoreductive surgery and intraperitoneal hyperthermic chemotherapy for peritoneal surface malignancy: experience with 501 procedures. J Am Coll Surg. 2007; 204(5): 943-953; discussion 53-5.

9. Gusani NJ, Cho SW, Colovos C, et al. Aggressive surgical management of peritoneal carcinomatosis with low mortality in a high-volume tertiary cancer center. Ann Surg Oncol. 2008; 15(3): 754-763.

10. Glehen O, Gilly FN, Boutitie F, et al. Toward curative treatment of peritoneal carcinomatosis from nonovarian origin by cytoreductive surgery combined with perioperative intraperitoneal chemotherapy: a multi-institutional study of 1,290 patients. Cancer. 2010; 116(24): 5608-5618.

11. Chua TC, Liauw W, Saxena A, et al. Evolution of locoregional treatment for peritoneal carcinomatosis: single-center experience of 308 procedures of cytoreductive surgery and perioperative intraperitoneal chemotherapy. Am J Surg. 2011; 201(2): 149-156.

12. Bonvalot S, Cavalcanti A, Le Péchoux C, et al. Randomized trial of cytoreduction followed by intraperitoneal chemotherapy versus cytoreduction alone in patients with peritoneal sarcomatosis. Eur J Surg Oncol. 2005; 31(8): 917-923.

13. Baumgartner JM, Ahrendt SA, Pingpank JF, et al. Aggressive locoregional management of recurrent peritoneal sarcomatosis. J Surg Oncol. 2013; 107(4): 329-334.

14. Sommariva A, Pasquali S, Del Fiore P, et al. Cytoreductive surgery and hyperthermic intraperitoneal chemotherapy in patients with peritoneal sarcomatosis: long-term outcome from a single institution experience. Anticancer Res. 2013; 33(9): 3989-3994.

15. Randle RW, Swett KR, Shen P, Stewart JH, Levine EA, Votanopoulos KI. Cytoreductive surgery with hyperthermic intraperitoneal chemotherapy in peritoneal sarcomatosis. Am Surg. 2013; 79(6): 620624.

16. Bryan ML, Fitzgerald NC, Levine EA, Shen P, Stewart $\mathrm{JH}$, Votanopoulos KI. Cytoreductive surgery with hyperthermic intraperitoneal chemotherapy in sarcomatosis from gastrointestinal stromal tumor. Am Surg. 2014; 80(9): 890-895.

17. Abu-Zaid A, Azzam A, Abuzaid M, et al. Cytoreductive surgery plus hyperthermic intraperitoneal chemotherapy for management of peritoneal sarcomatosis: A preliminary single-center experience from Saudi Arabia. Gastroenterol Res Pract. 2016; 2016: 6567473.

18. Karamveri C, Pallas N, Kyziridis D,et al. Cytoreductive Surgery in Combination with HIPEC in the Treatment of Peritoneal Sarcomatosis. Indian J Surg Oncol. 2019; 10(1): 40-45.

19. Dziewirski W, Rutkowski P, Nowecki ZI, et al. Surgery combined with intraoperative brachytherapy in the treatment of retroperitoneal sarcomas. Ann Surg Oncol. 2006; 13(2): 245-252.

20. Sweeting RS, Deal AM, Llaguna OH, et al. Intraoperative electron radiation therapy as an important treatment modality in retroperitoneal sarcoma. J Surg Res. 2013; 185(1): 245-249.

21. Roeder F, Ulrich A, Habl G, et al. Clinical phase I/II trial to investigate preoperative dose-escalated intensity-modulated radiation therapy (IMRT) and intraoperative radiation therapy (IORT) in patients with retroperitoneal soft tissue sarcoma: interim analysis. BMC Cancer. 2014; 14: 617. 
22. Stucky CC, Wasif N, Ashman JB, Pockaj BA, Gunderson LL, Gray RJ. Excellent local control with preoperative radiation therapy, surgical resection, and intra-operative electron radiation therapy for retroperitoneal sarcoma. J Surg Oncol. 2014; 109(8): 798-803.

23. Sindelar WF, Kinsella TJ, Chen PW, et al. Intraoperative radiotherapy in retroperitoneal sarcomas. Final results of a prospective, randomized, clinical trial. Arch Surg. 1993; 128(4): 402-410.

24. Ueda T, Aozasa $\mathrm{K}$, Tsujimoto $\mathrm{M}$, et al. Prognostic significance of Ki-67 reactivity in soft tissue sarcomas. Cancer.1989; 63(8): 1607-1611.

25. Liang Y, Wang W, Li Jet al. Combined use of the neutrophil-lymphocyte and platelet-lymphocyte ratios as a prognostic predictor in patients with operable soft tissue sarcoma. J Cancer. 2018; 9(12): 2132-2139.

26. Chan JY, Zhang Z, Chew W, et al. Biological significance and prognostic relevance of peripheral blood neutrophil-to-lymphocyte ratio in soft tissue sarcoma. Sci Rep. 2018; 8(1): 11959.

27. Que Y, Qiu H, Li Y, et al. Preoperative plateletlymphocyte ratio is superior to neutrophillymphocyte ratio as a prognostic factor for soft-tissue sarcoma. BMC Cancer. 2015; 15: 648.

28. Azzam AZ, Alyahya ZA, Wusaibie AAA, Amin TM. Cytoreductive surgery and hyperthermic intraperitoneal chemotherapy in the management of pseudomyxoma peritonei: A single-center experience. Indian J Gastroenterol. 2017; 36(6): 452 458.

29. Sugarbaker PH. Peritonectomy procedures. Ann Surg. 1995; 221(1): 29-42.

30. Zaki Azzam A, Alqarni A, Mahmoud Amin T. The role of intraoperative radiotherapy (IORT) in the management of patients with pancreatic and periampullary cancer: A single center experience. J Egypt Natl Canc Inst. 2018; 30(2): 77-79.

31. Azzam AZ, Amin TM. Combined intraoperative radiotherapy (IORT) and hyperthermic intraperitoneal chemotherapy (HIPEC) with cytoreduction surgery (CRS) as a novel approach in the management of resectable pancreatic cancer. Gulf J Oncol. 2020; 1(33): 19-26.

32. Dindo D, Demartines N, Clavien PA. Classification of surgical complications: a new proposal with evaluation in a cohort of 6336 patients and results of a survey. Anna Surg. 2004; 240(2): 205-213.

33. Jacquet P, Sugarbaker PH. Peritoneal-plasma barrier. Cancer Treat Res. 1996; 82: 53-63.

34. Flessner MF. The transport barrier in intraperitoneal therapy. Am J Physiol Renal Physiol. 2005; 288(3): F433-442.

35. Speyer JL, Sugarbaker PH, Collins JM, Dedrick RL, Klecker RW Jr, Myers CE. Portal levels and hepatic clearance of 5-fluorouracil after intraperitoneal administration in humans. Cancer Res. 1981; 41(5): 1916-1922.
36. Yan TD, Black D, Savady R, Sugarbaker PH. A systematic review on the efficacy of cytoreductive surgery and perioperative intraperitoneal chemotherapy for pseudomyxoma peritonei. Ann Surg Oncol. 2007; 14(2): 484-492.

37. Yan TD, Welch L, Black D, Sugarbaker PH. A systematic review on the efficacy of cytoreductive surgery combined with perioperative intraperitoneal chemotherapy for diffuse malignancy peritoneal mesothelioma. Ann Oncol. 2007; 18(5): 827-834.

38. Yan TD, Black D, Savady R, Sugarbaker PH. Systematic review on the efficacy of cytoreductive surgery combined with perioperative intraperitoneal chemotherapy for peritoneal carcinomatosis from colorectal carcinoma. J Clin Oncol. 2006; 24(24): 40114019.

39. Yonemura Y, Bando E, Kawamura $\mathrm{T}$, et al. Cytoreduction and intraperitoneal chemotherapy for carcinomatosis from gastric cancer. Cancer Treat Res. 2007; 134: 357-373.

40. Al-Badawi IA, Abu-Zaid A, Azzam A, AlOmar O, AlHusaini H, Amin T. Cytoreductive surgery and hyperthermic intraperitoneal chemotherapy for management of recurrent/relapsed ovarian granulosa cell tumor: a single-center experience. J Obstet Gynaecol Res. 2014; 40(9): 2066-2075.

41. Sugarbaker P, Ihemelandu C, Bijelic L. Cytoreductive surgery and HIPEC as a treatment option for laparoscopic resection of uterine leiomyosarcoma with morcellation: Early results. Ann Surg Oncol. 2016; 23(5): 1501-1507.

42. Sugarbaker PH, Ryan DP. Cytoreductive surgery plus hyperthermic perioperative chemotherapy to treat peritoneal metastases from colorectal cancer: standard of care or an experimental approach? Lancet Oncol. 2012; 13(8): e362-369.

43. Baratti D, Pennacchioli E, Kusamura S, et al. Peritoneal sarcomatosis: is there a subset of patients who may benefit from cytoreductive surgery and hyperthermic intraperitoneal chemotherapy? Ann Surg Oncol. 2010; 17(12): 3220-3228.

44. Khomyakov V, Ryabov A, Ivanov A, et al. Bidirectional chemotherapy in gastric cancer with peritoneal metastasis combining intravenous XELOX with intraperitoneal chemotherapy with low-dose cisplatin and Doxorubicin administered as a pressurized aerosol: an open-label, Phase-2 study (PIPAC-GA2). Pleura Peritoneum. 2016; 1(3): 159-166.

45. Mehta AM, Huitema AD, Burger JW, Brandt-Kerkhof AR, van den Heuvel SF, Verwaal VJ. Standard clinical protocol for bidirectional hyperthermic intraperitoneal chemotherapy (HIPEC): systemic leucovorin, 5-fluorouracil, and heated intraperitoneal oxaliplatin in a chloride-containing carrier solution. Ann Surg Oncol. 2017; 24(4): 990-997.

46. Hakeam H, Ayman A, Waleed AT, Amen T. Systemic complications of the bidirectional intraoperative chemotherapy with intravenous ifosfamide and hyperthermic intraperitoneal chemotherapy (HIPEC) 
using cisplatin plus doxorubicin. Pleura Peritoneum. 2019; 4(4): 20190025.

47. Naffouje SA, Tulla KA, Salti GI. A Simplified Peritoneal Sarcomatosis Score for patients treated with cytoreductive surgery and hyperthermic intraperitoneal chemotherapy. J Gastrointest Oncol. 2018; 9(6): 1138-1143.

48. Jacquet P, Sugarbaker PH. Clinical research methodologies in diagnosis and staging of patients with peritoneal carcinomatosis. Cancer Treat Res. 1996; 82: 359-374.

49. Bhatt A, Yonemura Y, Mehta S, et al. The pathologic peritoneal cancer index (PCI) strongly differs from the surgical PCI in peritoneal metastases arising from various primary tumors. Ann Surg Oncol. 2020; 27(8): 2985-2996.

50. Berger Y, Jacoby H., Kaufmann MI, et al. Correlation between intraoperative and pathological findings for patients undergoing cytoreductive surgery and hyperthermic intraperitoneal chemotherapy. Ann Surg Oncol. 2019; 26(4): 1103-1109.

51. Hasegawa T, Yamamoto S, Yokoyama R, Umeda T, Matsuno Y, Hirohashi S. Prognostic significance of grading and staging systems using MIB-1 score in adult patients with soft tissue sarcoma of the extremities and trunk. Cancer. 2002; 95(4): 843-851.
52. Azzam AZ, Dababo MA, Azzam KA, Almeshal M, Amin TM. Right temporal brain metastases arising three years after curative resection of gastric cancer: A case report. Gulf J Oncol. 2018; 1(28): 75-77.

53. Al Harbi TZ, Azzam KA, Azzam A, Amin T, Bakshi N. Incidentally discovered persistent mullerian duct syndrome in a 45-year-old male presenting with germ cell tumor and bilateral cryptorchidism: A rare case report and review of the literature. Int J Surgery Case Rep. 2018; 43: 41-44.

54. Klaver YL, Lemmens VE, Nienhuijs SW, Nieuwenhuijzen GA, Rutten HJ, de Hingh IH. Intraoperative radiotherapy and cytoreductive surgery with hyperthermic intraperitoneal chemotherapy. Five consecutive case reports of locally advanced rectal cancer with synchronous peritoneal carcinomatosis. Strahlenther Onkol. 2013; 189(3): 256-260.

55. Azzam AZ, Al-Hamadah FH, Azzam KA, Amin TM. Mucinous appendiceal adenocarcinoma penetrated the quadratus lumborum muscle posteriorly and presented as gluteal abscess managed aggressively with drainage followed by cytoreductive surgery and hyperthermic intraperitoneal chemotherapy combined with intraoperative radiotherapy. A case report and review of the literature. Indian J Surg Oncol. 2020; 11(Suppl 1): 128-130. 